\title{
Symbolic control of attention: Tracking its temporal dynamics
}

\author{
BERNHARD HOMMEL \\ Leiden University, Leiden, The Netherlands \\ AND \\ ELKAN G. AKYÜREK \\ Ludwig Maximilian University, Munich, Germany
}

\begin{abstract}
Three experiments examined the temporal dynamics of the impact of symbols with task-irrelevant spatial meanings on attentional control. In Experiments 1 and 2, participants were color-cued to report the first letter they saw in the left or right of two parallel letter streams. The cue appeared in the shape of an arrow pointing to the target stream (compatible) or to the other stream (incompatible). Incompatible arrows delayed letter selection; that is, participants reported later-appearing letters and made more errors. In Experiment 3, the target stream was cued in advance, and yet, incompatible symbols delayed target selection. These findings suggest that the irrelevant meaning of symbolic stimuli can still penetrate and bias attentional top-down control, an observation that challenges available control theories.
\end{abstract}

Human communication is based on the exchange of symbols in order to produce intended modifications of an addressee's cognitive state and behavior (Grice, 1969). Symbols can thus be considered an important means to "direct one another's attention to particular aspects of their shared world" (Tomasello \& Call, 1997, p. 408). Indeed, there is ample evidence that pointing gestures, directional signs (such as arrows), and even directional words (such as "left" and "right") direct people's attention toward the location they point or refer to (e.g., Hommel, Pratt, Colzato, \& Godijn, 2001; Langton \& Bruce, 2000). Interestingly, this is true not only for the rather trivial case when such symbols are functional and meaningful for the current task (e.g., when searching for directions), but also when their meaning is entirely irrelevant and uninformative.

In the context of communication, this makes a lot of sense, because a message to a receiver should communicate its meaning even if it does not fully meet all aspects of the receiver's current activities and goals-otherwise, e-mail reminders simply would not work. However, attentional approaches have often overlooked the automatic attentional impact of directional symbols. Indeed, common wisdom since the pioneering work of Posner (1980) and Jonides $(1980,1981)$ has taken it for granted that so-called peripheral or exogenous spatial cues (cues that appear at the same location as the cued event) attract attention in an automatic fashion, whereas central or endogenous cues (cues that appear at a spatially neutral location but indicate the location of the cued event) require the observer to actively translate the information provided by the cue into a voluntary shift of the attentional focus. Findings suggesting that stimuli can not only attract attention (to their location) but also direct attention (to other locations) undermine the intuitive distinction between exogenous and endogenous cues, because it seems that spatially meaningful symbols can take over direct attentional control, especially if they are deictic in nature (Gibson \& Kingstone, 2006). More concretely, activating the representations of spatial symbols seems to induce a top-down bias of attention toward selecting stimuli that share the feature indicated by the symbol - such as a particular location (Pratt \& Hommel, 2003; see Figure 1).

The present study aimed at exploring the temporal dynamics of symbolic control. Temporal characteristics of cuing are commonly investigated by manipulating the interval between cue presentation and target presentation. Previous manipulations of this sort have shown that, if the interval is very short, cuing effects are small or absent, presumably because it takes some time for the cue to be encoded (see, e.g., Müller \& Rabbitt, 1989) and for its impact on control processes to unfold (Pratt \& Hommel, 2003). In contrast, once a robust cuing effect is obtained, extending the interval has no effect (Hommel et al., 2001; Pratt \& Hommel, 2003). Interestingly, this temporal pattern is different from what has been reported for peripheral cues (e.g., Müller \& Rabbitt, 1989; Posner \& Cohen, 1984), which induce fast but transient attentional biases. Along the lines of Pratt and Hommel, this dissociation is likely to suggest that peripheral cues trigger a shift of the 


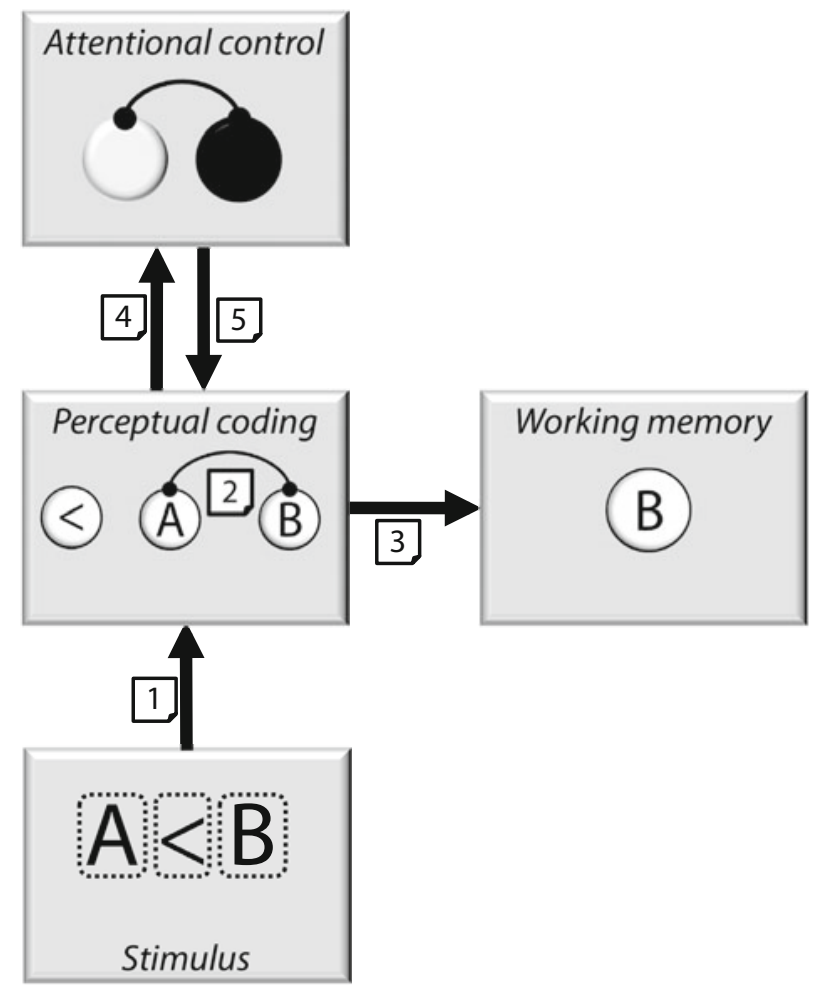

Figure 1. Application of Pratt and Hommel's (2003) account of symbolic cuing effects to the present Experiment 1. In the example, participants have to select one of two stimulus letters ( $A$ and $B$, in the example) on the basis of the color (but not the direction) of a central arrow and the target location it indicates. In the example, this color (not shown) is green, indicating that the right letter is the target. (1) The letters, the color, and the direction of the arrow are encoded at a perceptual coding stage. Stimulus representations are integrated and thus contain all features of a given stimulus (e.g., identities and locations of letters, color and direction of arrow). (2) The codes of the letters A and B compete and inhibit each other because they are alternative targets. (3) The letter code that wins the competition is consolidated into working memory (the letter B, in this case). (4) Information about the color of the arrow is transmitted to an attentional control system that (5) biases competition at the perceptual encoding stage toward goal-relevant stimuli (targets). Because the color indicates that the target location is "right," the corresponding < right > control node is activated. However, given that the direction of the arrow also provides spatial information, it activates the corresponding control node (the <left> node, in the example). Control nodes compete for control, so that activating the $<$ left $>$ node will slow down the action of the <right $>$ node. Hence, the top-down bias favoring right stimuli (i.e., stimuli, the integrated representation of which contains the feature < right $>$ ) is delayed or temporarily weakened by the presence of a left-pointing arrow and the competition it creates at the attentional control stage.

attentional focus, whereas symbolic cues produce a competition bias. Assuming that focus shifts induced by irrelevant stimuli are more likely to be undone immediately than are selection biases, this explains why symbolic cues affect attention over a longer period of time.

The available evidence from manipulations of the cuetarget interval thus suggests that cue-induced effects on attentional control are temporally rather stable. The present study used a different approach in order to assess the functional consequences of cue processing, and the temporal characteristics thereof, more directly.

\section{EXPERIMENT 1}

In Experiment 1, participants were presented with two spatially distinct but concurrent streams of randomly chosen letters (see Figure 2). Halfway through the stream, a color cue would appear, probing the participant to report the next upcoming letter from the left or the right stream. Reporting a letter therefore required encoding and interpreting the cue and shifting attention toward the cued stream. These operations take some time to complete, so that participants were expected to miss some of the letters presented since cue onset. The number of letters missed is a direct reflection of the time demands of these operations, so that it can be used to compute what Weichselgartner and Sperling (1987) called attention reaction time (ART = lag of letter reported $\times$ presentation time per letter).

More interesting than the time demands of these processes was the way these demands would be affected by the task-irrelevant spatial meaning of symbols. As indicated in Figure 2, the color cues had the shape of arrows pointing to the left or right. The direction in which they pointed was irrelevant and uninformative, and participants were encouraged to ignore it. Nevertheless, given the observations of Hommel et al. (2001) and Pratt and Hommel (2003), we expected that the arrows would bias visual attention toward the locations they pointed to. This should affect performance in trials in which the arrow pointed to the wrong stream - that is, when the color called for the selection of a target from one stream while the arrow pointed to the other stream. This was expected to have two possible consequences. For one, participants might be able to report a letter from the correct stream but would need more time to do so, because biasing attentional control accordingly would be counteracted by the symbolic cue. Theoretically speaking, driving the appropriate control node (see Figure 1) above threshold would take longer in the face of competing activation induced by the incompatible spatial symbol. This should increase ART; that is, participants should tend to report letters with a longer lag. Second, participants might sometimes be unable to overcome the bias induced by the symbol, and therefore bias attention toward the wrong stream. If so, they should report a letter from this incorrect stream, and they should be more likely to do so in trials in which the color and the arrow indicate two different streams.

\section{Method}

Eight students participated for pay in single sessions of about $30 \mathrm{~min}$. Display and timing were controlled to the nearest millisecond by a standard PC. Stimuli were presented at three locations, one about $0.5^{\circ}$ of visual angle (viewing distance about $60 \mathrm{~cm}$ ) to the left of the screen center, one exactly at screen center, and one $0.5^{\circ}$ to the right of the screen center. The left and the right location were used to present two letter sequences, each one containing 14 uppercase letters appearing in white on the black background. The 14 letters making up the first seven frames of the two sequences were randomly selected (without replacement) from the ASCII alphabet, from which the letters G, J, N, Q, V, X, and Y were excluded, to avoid feature confu- 


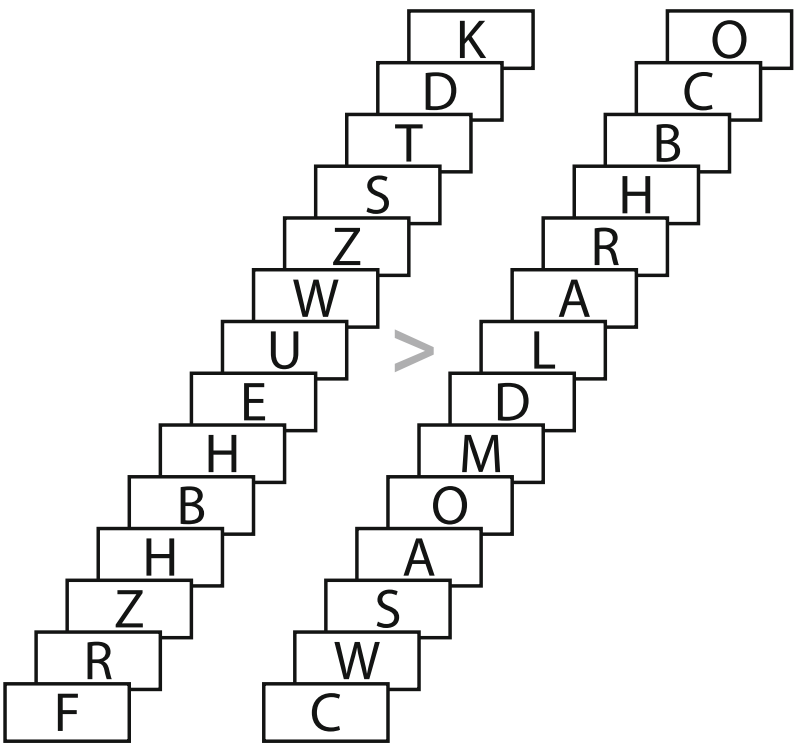

Figure 2. Sequence of events in Experiment 1. Presentation time per frame was 157 msec. The centrally presented cue was blue or green, and it stayed until the end of the trial.

sions. The remaining 14 letters, which made up the remaining seven frames of the two sequences, were determined the same way. Hence, no letter could appear more than once during the first seven or the last seven frames of both letter streams, and no letter could appear more than twice during a whole trial. ${ }^{1}$ The central location was exclusively used to present the cue that indicated the target stream. It always appeared at the eighth frame and consisted of a left- or right-pointing arrow $(<,>)$ appearing in blue or green, with the color indicating the location of the target stream. All stimuli were presented in DOS text mode; each symbol measured about $0.3^{\circ}$ in width and $0.4^{\circ}$ in height. Participants responded with their dominant hand by pressing the corresponding letter key on the computer keyboard.

After an intertrial interval of $3 \mathrm{sec}$, each trial began with the presentation of the two letter streams - that is, of 14 pairs of letters, each pair being replaced by the next after $157 \mathrm{msec}$. Simultaneously with the eighth letter pair, the target cue was presented at the center, where it stayed until the presentation of the last pair. The color of the cue indicated whether participants were to respond from the left or the right stream. The blue and green colors indicated the left and right streams, respectively, for half of the participants, whereas the other half received the opposite color-to-location mapping. After the last pair was presented, participants were prompted to type in, at their leisure, the first letter they recognized in the cued stream after cue presentation. Each color was used on a left- or right-pointing arrow in $50 \%$ of the trials; hence, the correlation between color and arrow direction was zero. This was pointed out to the participants before the experiment, and they were instructed to therefore ignore the arrow directions entirely. There were four experimental conditions resulting from the combinations of left and right target streams and left- and right-pointing arrows as irrelevant features of the cue. Each participant first performed 12 practice trials -3 replications of each of the four conditions - and then worked through 160 experimental trials - 40 replications of each of the four conditions. Conditions were ordered randomly, except that conditions were not allowed to occur more often than three times in a row.

\section{Results and Discussion}

Responses fell into three categories. First, the reported letter may have been one of the last 7 letters (i.e., from color cue onset on) presented in the cued stream. In this case, the lag of the reported letter with respect to the cue was calculated (lag 0 referring to the letter appearing at the same time as the cue). Second, the reported letter may have been one of the last 7 letters from the wrong, uncued stream, which was considered an error. Third, the reported letter may not have been among the 14 letters presented from color cue onset on, in which case the response was considered a miss.

The reliability criterion for all analyses was $p<.05$. Misses accounted for $0.9 \%$ of the trials, and were not analyzed further. Lags of correct reports underwent an ANOVA for repeated measures, with correct stream location and arrow direction as independent variables. There were no main effects, but the interaction was reliable $\left[F(1,7)=40.00, M S_{\mathrm{e}}=.06, p<.001\right]$. As shown in Table 1, lags were shorter if the arrow pointed to the correct stream than if it did not. With regard to ART, this effect indicates that arrows that were incompatible with the required shift of the attentional bias prolonged it by $83 \mathrm{msec}$ (408 msec for incompatible arrows minus $325 \mathrm{msec}$ for compatible ones). Finally, incompatible arrows also increased the percentage of errors (see Table 1), but an ANOVA on error rates yielded only a close-to-significant interaction $\left[F(1,7)=4.62, M S_{\mathrm{e}}=25.25, p<.07\right]$.

The outcome is clear-cut: Biasing attention toward left or right stimuli is hampered by symbols that refer to or "point" (in a metaphorical sense) in the opposite direction. The costs produced by incompatible symbols are substantial, as the 83-msec increase of ART indicates. The error rates show the same pattern, but the overall rate was not excessive and the compatibility effect mild, suggesting that participants were mostly able to eventually overcome the arrow-induced bias. Together with previous observations of Hommel et al. (2001) and Pratt and Hommel (2003), the outcome of the present Experiment 1 suggests three preliminary conclusions. First, the arrow stimuli seem to affect performance automatically - that is, despite the fact that arrow directions were uninformative, irrelevant to the task, and to be ignored.

Second, the way the arrows affect performance is different from their role in the Stroop-like effects that arrows have been reported to produce. The classical effects fall into two categories. One indicates conflict originating from the incongruence between two stimulus features, the

Table 1

Experiment 1: Mean Lags of Correctly Reported Letters (M), Together With Standard Deviations (SDs) and Attentional Reaction Time (ART), and Mean Percent Incorrectly Reported Letters (From the Wrong Stream), Together With $S D$ s, As a Function of Target-Stream Location and Direction of Arrow

\begin{tabular}{|c|c|c|c|c|c|c|}
\hline \multirow{2}{*}{$\begin{array}{l}\text { Target } \\
\text { Stream }\end{array}$} & \multicolumn{3}{|c|}{ Left Arrow } & \multicolumn{3}{|c|}{ Right Arrow } \\
\hline & $M$ & $S D$ & ART & $M$ & $S D$ & $\overline{\text { ART }}$ \\
\hline & \multicolumn{6}{|c|}{ Lag Correct Report } \\
\hline Left & 2.01 & 0.4 & 316 & 2.51 & 0.4 & 394 \\
\hline Right & 2.68 & 0.6 & 421 & 2.13 & 0.5 & 334 \\
\hline \multicolumn{7}{|c|}{ Percent Incorrect Report } \\
\hline Left & 3.16 & 4.9 & & 5.72 & 5.8 & \\
\hline Right & 7.02 & 5.1 & & 1.94 & 3.6 & \\
\hline
\end{tabular}


direction of an arrow and another spatial feature, such as the location in which the arrow appears. For instance, responding to the location of a target stimulus appearing at the top or bottom of a screen takes longer if the stimulus at the top consists of an arrow pointing down or if the stimulus at the bottom consists of an arrow pointing up (Clark \& Brownell, 1975; Logan, 1980). Effects falling into the other category indicate conflict originating from stimulus-response incompatibility. For instance, carrying out left and right responses to the color of a target stimulus takes longer if that color comes in the shape of an arrow pointing in the "wrong" direction (for an overview, see $\mathrm{Lu}$ \& Proctor, 1995). Both types of effects are consistent with our findings in showing that arrows can affect information processing in unintentional ways, but they differ in the way they do so. Effects of stimulus-stimulus incongruence and stimulus-response incompatibility are assumed to result from conflict between stimulus-induced response tendencies (e.g., Kornblum, Hasbroucq, \& Osman, 1990; $\mathrm{Lu} \&$ Proctor, 1995), which is unlikely to account for our present findings. Rather than priming responses, the irrelevant meaning of the arrows we presented seemed to have primed attentional control operations - that is, cognitive operations that serve to bias attentional selection toward particular stimuli (stimuli appearing on the left or right, in our case).

Third, our findings suggest that carrying out such a control operation takes about $300-400 \mathrm{msec}$ and that operation-incongruent arrows (i.e., arrows priming a conflicting operation) prolong the execution of the operation by about $80 \mathrm{msec}$. The first part of the observation fits well with the reports of Reeves and Sperling (1986) and Weichselgartner and Sperling (1987), who estimated the time to identify (the stimulus signaling) the need for a shift of spatial attention and to program and perform the shift to be about $300 \pm 100 \mathrm{msec}$. Considering this number, the delay of $83 \mathrm{msec}$ seems quite substantial, especially given that the direction and meaning of the arrow were entirely task irrelevant. However, there are reasons to assume that aspects of our task led to an overestimation of this delay, a possibility that we investigated in Experiment 2.

\section{EXPERIMENT 2}

In Experiment 1, the stream from which the target was to be selected was indicated by the color of the central cue. To make efficient use of this cue required quite a bit of cognitive resources, because the participant needed to hold the arbitrary color-location mapping in mind, to identify the color of the cue, and to apply the memorized mapping to derive the relevant stream location. The literature on various kinds of stimulus-response compatibility suggests that nominally irrelevant information is more likely to intrude, the more complex the task is and, more specifically, the more complex and arbitrary the relevant stimulus-response transformations are (e.g., Glaser \& Glaser, 1989; Lu, 1997; Virzi \& Egeth, 1985). From that perspective, signaling stream location by color must have rendered the task rather difficult and may have made it particularly vulnerable to the impact of irrelevant stimuli.
To see whether this impact can still be observed if the relevant stream is cued in a more straightforward and less arbitrary fashion, we used the same color cues in Experiment 2 but colored the streams they cued, as well. That is, we replicated Experiment 1 but presented the left and right stream in blue or green (or vice versa), so that the color of the cue would always match the stream it indicated. Accordingly, participants no longer needed to memorize any color-location rule and did not even need to identify the color of the cue; a perceptual match was fully sufficient. Moreover, given that cue and stream elements had the same color, they appeared as one visual group, which should also have facilitated target selection.

\section{Method}

Sixteen students participated for pay. The method was as in Experiment 1 , except that the letters appearing in one stream were all green and the letters in the other stream were all blue (the mapping of colors to stream locations was constant for a given participant but balanced across participants). The color of the target cue always matched the color of the stream it signaled.

\section{Results and Discussion}

Misses accounted for $4.5 \%$ of all trials. The ANOVA of lags produced no main effect but a reliable interaction $\left[F(1,15)=4.71, M S_{\mathrm{e}}=.05, p<.05\right]$. As shown in Table 2, lags were shorter if the arrow pointed to the correct stream than if it did not. Again, this indicated that ART was longer for incompatible than for compatible arrows (394 vs. $374 \mathrm{msec}$ ), which amounts to a compatibilityrelated delay of $20 \mathrm{msec}$. An ANOVA that included the data from Experiment 1 yielded a three-way interaction $\left[F(1,22)=15.76, M S_{\mathrm{e}}=.05, p<.001\right]$, which confirms that the compatibility effect was larger in Experiment 1 than in Experiment 2. The ANOVA of the error rates did not yield reliable effects.

The outcome is clear in suggesting two conclusions. First, simplifying the task and the mapping between cue and target location drastically reduced the processing delay created by the irrelevant spatial meaning of the arrows. ${ }^{2}$ This supports previous observations that tasks that rely on arbitrary stimulus-response rules are more vulnerable to distracting stimuli (Glaser \& Glaser, 1989; Lu, 1997; Virzi \& Egeth, 1985) and justifies our suspicion that the delay obtained in Experiment 1 was overestimated.

Table 2

Experiment 2: Mean Lags of Correctly Reported Letters ( $M)$, Together With Standard Deviations (SDs) and Attentional

Reaction Time (ART), and Mean Percent Incorrectly Reported Letters (From the Wrong Stream), Together With SDs, As a Function of Target-Stream Location and Direction of Arrow

\begin{tabular}{|c|c|c|c|c|c|c|}
\hline \multirow{2}{*}{$\begin{array}{l}\text { Target } \\
\text { Stream }\end{array}$} & \multicolumn{3}{|c|}{ Left Arrow } & \multicolumn{3}{|c|}{ Right Arrow } \\
\hline & $M$ & $S D$ & ART & $M$ & $S D$ & ART \\
\hline & \multicolumn{6}{|c|}{ Lag Correct Report } \\
\hline Left & 0.52 & 0.6 & 82 & 0.64 & 0.7 & 100 \\
\hline Right & 0.64 & 0.8 & 100 & 0.55 & 0.7 & 86 \\
\hline \multicolumn{7}{|c|}{ Percent Incorrect Report } \\
\hline Left & 4.57 & 10.1 & & 6.16 & 9.5 & \\
\hline Right & 7.25 & 9.5 & & 5.74 & 12.4 & \\
\hline
\end{tabular}


Second, however, even with highly intuitive cues that must have made it much easier to ignore the arrows and their direction, we found reliable processing delays. Hence, the arrows still biased attentional control operations to a detectable degree.

\section{EXPERIMENT 3}

In Experiment 1, the spatial symbol was presented at the same time as the relevant color cue. This suggests that the symbol and the cue competed for controlling attentionthat is, for activating control nodes that provide top-down support for stimuli presented in the left or right stream. It is possible that the impact of irrelevant information is restricted to situations in which control systems are in a kind of "selection mode" - that is, when they are in the process of settling into a new control state. For instance, Waszak, Hommel, and Allport $(2003,2005)$ observed that stimuli that have been associated with a particular task tend to activate this task automatically when they are encountered later on. However, this effect was mainly restricted to situations in which people were to switch to a new task, but not if the task was repeated. Applied to the present context, this may imply that the symbolic stimuli can affect attentional control only if a new control state has to be established. If so, arrows should be ineffective if participants know in advance from which visual stream the target will need to be selected. This hypothesis was tested in Experiment 3, which replicated Experiment 1 except that the relevant stimulus stream was cued in advance. Accordingly, participants were able to bias their control system long before the target stimuli appeared. The (compatible or incompatible) arrow still appeared to indicate when the target stimuli were presented, but it no longer varied in color.

\section{Method}

Eighteen students participated for pay. The method was as in Experiment 1 , with the following exceptions. Before the two stimulus streams were presented, the relevant stream was cued. The locations of the two streams were indicated by two minus signs to the left and right of a central fixation cross. Two hundred milliseconds after the three symbols appeared on the screen, one of the two minus signs was replaced by an asterisk for $1 \mathrm{sec}$, thus cuing the left or right stream. Then the asterisk turned into a minus sign, and $100 \mathrm{msec}$ later the trial proceeded as in Experiment 1. The central arrow was always green.

\section{Results and Discussion}

Misses accounted for $3.1 \%$ of all trials. The ANOVA of lags produced no main effect but a reliable interaction $\left[F(1,17)=11.28, M S_{\mathrm{e}}=.02, p<.005\right]$. As shown in Table 3, lags were shorter if the arrow pointed to the correct stream than if it did not. Again, this indicated that ART was longer for incompatible than for compatible arrows (100 vs. $84 \mathrm{msec}$ ), which amounts to a compatibilityrelated delay of $16 \mathrm{msec}$. An ANOVA on the data from Experiments 2 and 3 did not produce a reliable three-way interaction $[F(1,32)<1]$, indicating that the compatibility effects in the two experiments are equivalent. The ANOVA of the error rates did not yield reliable effects.
Table 3

Experiment 3: Mean Lags of Correctly Reported Letters $(M)$, Together With Standard Deviations (SDs) and Attentional

Reaction Time (ART), and Mean Percent Incorrectly Reported

Letters (From the Wrong Stream), Together With $S D$ s, As a

Function of Target-Stream Location and Direction of Arrow

\begin{tabular}{|c|c|c|c|c|c|c|}
\hline \multirow{2}{*}{$\begin{array}{c}\text { Target } \\
\text { Stream }\end{array}$} & \multicolumn{3}{|c|}{ Left Arrow } & \multicolumn{3}{|c|}{ Right Arrow } \\
\hline & $M$ & $S D$ & ART & $M$ & $S D$ & ART \\
\hline & \multicolumn{6}{|c|}{ Lag Correct Report } \\
\hline Left & 2.37 & 0.7 & 372 & 2.52 & 0.6 & 396 \\
\hline Right & 2.49 & 0.5 & 391 & 2.38 & 0.5 & 374 \\
\hline \multicolumn{7}{|c|}{ Percent Incorrect Report } \\
\hline Left & 7.6 & 9.7 & & 10.8 & 13.8 & \\
\hline Right & 10.2 & 12.8 & & 10.1 & 15.7 & \\
\hline
\end{tabular}

The observation that cuing the relevant stream in advance does not reliably reduce the compatibility effect provides no evidence that the impact of spatial symbols on attentional control will be restricted to situations in which new control parameters are to be established. This is not to say that the impact of arrows is independent of the task as such, an issue we will get back to in the General Discussion. And yet, it does not seem as if there would be particular phases in the preparation of an attentional operation that would be immune to the impact of an arrow.

\section{GENERAL DISCUSSION}

This study aimed at assessing the functional consequences of symbolic cuing for attentional control, as well as the temporal characteristics of these consequences. Taken together, the three experiments confirm that symbols with spatial meaning affect attentional control automatically - that is, even if considering them is of no use. The experiments further demonstrate that control is affected whether the cue is difficult (Experiment 1) or easy (in Experiment 2) to interpret and whether establishing a new control parameter is under way (Experiments 1 and 2) or has been completed already (Experiment 3).

Especially the observation that symbols affect attentional control even after the system has settled into a new control state (as in Experiment 3) calls for modifications of available control theories. Current models of attention and action control are decidedly top-down oriented, assuming that the communication between control processes and the processes being controlled is a one-way street. For instance, Logan and Gordon's (2001) executive control theory of visual attention (ECTVA), which is an attempt to integrate Bundesen's (1990) theory of visual attention with considerations about attentional and actioncontrol operations, claims that control is exerted in three steps: (1) The task instruction is held in working memory; (2) the instructions are translated into a set of control parameters, with the attentional bias being one of them; and (3) these parameters are used to modulate information processing so as to achieve the task goal. Importantly, the control parameters are specified and implemented in a purely top-down fashion, with no way for bottom-up 
information (apart from the instructions given) to have an impact on control processes - a design feature that ECTVA shares with other cognitive-control frameworks (e.g., Brown, Reynolds, \& Braver, 2007; Gilbert \& Shallice, 2002; Meiran, 2000; Meyer \& Kieras, 1997). From this perspective, it is difficult to explain symbolic cuing processes in general, but it seems particularly difficult to understand why symbols affect attentional control even after most control parameters (apart from the time point of information intake) have been specified.

It seems more likely that control is more dynamic and sensitive to ongoing competition. Consider the approach sketched in Figure 1. Implementing a control parameter may be thought of as priming one of the control nodes (i.e., increasing that node's activation), which again biases selection processes toward stimuli appearing at the corresponding location. Control states are known to be transient and labile (Altmann, 2002; Altmann \& Gray, 2002; Atkinson \& Birch, 1970), so that the activation of control nodes is effortful and may thus often be suboptimal. In other words, specifying a control parameter does not make it immune to competition-just as already selected responses are not immune to the impact of incompatible stimuli (Hommel, 1996). Spatial symbols would prime their corresponding control node, and they would do so at any time they are presented. Accordingly, symbols with incompatible spatial meanings would lead to conflict with "intentional" control of attention; intentionally activated and symbol-activated control nodes would compete for biasing target selection. According to our observations, this conflict is measurable but not necessarily immenseas the estimates of 16-20 msec from Experiments 2 and 3 suggest - but may become quite dramatic, as under the less favorable conditions tested in Experiment 1.

This proposed scenario suggests that task-irrelevant stimulus attributes can directly impact cognitive control processes in a more or less automatic fashion. It is important to point out, however, that this is not to say that action control is stimulus driven. To begin with, all that the arrows could achieve was to bias already prepared control operations in one or another direction but not to trigger a new, task-independent operation. Numerous findings suggest that preparing for a task entails some sort of selfautomatization, in the sense that people configure their cognitive system in such a way that the occurrence of a particular stimulus event is sufficient to trigger a whole set of cognitive processes that eventually lead to the intended action goal (Bargh \& Ferguson, 2000; Hommel, 2000). Apparently, the spatial information provided by the arrows was able to trigger (as in error trials) or prime (as in correct but delayed trials) those processes that were responsible for target selection; and yet, our findings do not suggest that this would have been possible without active preparation of the processes that were primed or triggered. Moreover, even though the arrows themselves were not relevant or informative, they always appeared as part of a stimulus event that did have some use - be it that their color indicated the target stream (as in Experiments 1 and 2) or that the time point of their appearance indicated the presence of the target (as in Experiment 3). Hence, even though it is fair to say that processing of the arrows and their impact on attentional control were automatic, this automaticity depended on the task and the action goal, and can thus be considered an example of "conditional automaticity" in the sense of Bargh (1989).

Applied to human communication, our findings suggest that involuntarily received messages can indeed redirect the attention of the receiver if they contain the "proper keywords"- that is, cues that are related to information that the receiver has previously enabled to attract his or her attention. And yet, most messages will be unlikely to trigger the respective control operations all by themselves, which means that they can bias only the already willing.

\section{AUTHOR NOTE}

We thank Roberto Dell'Acqua, Asher Cohen, and an anonymous reviewer for helpful comments on a previous version of this article. B.H. is affiliated with the Institute for Psychological Research and the Leiden Institute for Brain and Cognition, Leiden University, Leiden, The Netherlands. Correspondence concerning this article should be addressed to B. Hommel, Leiden University, Department of Psychology, Cognitive Psychology Unit, Wassenaarseweg 52, 2333 AK Leiden, The Netherlands (e-mail: hommel@fsw.leidenuniv.nl).

\section{REFERENCES}

Altmann, E. M. (2002). Functional decay of memory for tasks. Psychological Research, 66, 287-297.

Altmann, E. M., \& Gray, W. D. (2002). Forgetting to remember: The functional relationship of decay and interference. Psychological Science, 13, 27-33.

AtKinson, J. W., \& Birch, D. (1970). The dynamics of action. New York: Wiley.

BARGH, J. A. (1989). Conditional automaticity: Varieties of automatic influence in social perception and cognition. In J. S. Uleman \& J. A. Bargh (Eds.), Unintended thought (pp. 3-51). London: Guilford.

Bargh, J. A., \& Ferguson, M. L. (2000). Beyond behaviorism: On the automaticity of higher mental processes. Psychological Bulletin, 126, 925-945.

Brown, J. W., Reynolds, J. R., \& Braver, T. S. (2007). A computational neural model of fractionated conflict-control mechanisms in task switching. Cognitive Psychology, 55, 37-85.

Bundesen, C. (1990). A theory of visual attention. Psychological Review, 97, 523-547.

Clark, H. H., \& Brownell, H. H. (1975). Judging up and down. Journal of Experimental Psychology: Human Perception \& Performance, 1, 339-352.

Gibson, B. S., \& Kingstone, A. (2006). Visual attention and the semantics of space: Beyond central and peripheral cues. Psychological Science, 17, 622-627.

Gilbert, S. J., \& Shallice, T. (2002). Task switching: A PDP model. Cognitive Psychology, 44, 297-337.

Glaser, W. R., \& Glaser, M. O. (1989). Context effects in Stroop-like word and picture processing. Journal of Experimental Psychology: General, 118, 13-42.

Grice, H. P. (1969). Utterer's meaning and intentions. Philosophical Review, 78, 147-177.

Hommel, B. (1996). S-R compatibility effects without response uncertainty. Quarterly Journal of Experimental Psychology, 49A, 546-571

Hommel, B. (2000). The prepared reflex: Automaticity and control in stimulus-response translation. In S. Monsell \& J. Driver (Eds.), Control of cognitive processes: Attention and performance XVIII (pp. 247-273). Cambridge, MA: MIT Press.

Hommel, B., Pratt, J., Colzato, L., \& Godisn, R. (2001). Symbolic control of visual attention. Psychological Science, 12, 360-365.

Jonides, J. (1980). Toward a model of the mind's eye's movement. Canadian Journal of Psychology, 34, 103-112.

JoNIDES, J. (1981). Voluntary versus automatic control over the mind's 
eye's movement. In J. [B.] Long \& A. [D.] Baddeley (Eds.), Attention and performance $I X$ (pp. 187-203). Hillsdale, NJ: Erlbaum.

Kornblum, S., HasbroucQ, T., \& Osman, A. (1990). Dimensional overlap: Cognitive basis for stimulus-response compatibility-A model and taxonomy. Psychological Review, 97, 253-270.

LANGTon, S. R. H., \& BRUCE, V. (2000). You must see the point: Automatic processing of cues to the direction of social attention. Journal of Experimental Psychology: Human Perception \& Performance, 26, 747-757.

Logan, G. D. (1980). Attention and automaticity in Stroop and priming tasks: Theory and data. Cognitive Psychology, 12, 523-553.

LOGAN, G. D., \& GoRdON, R. D. (2001). Executive control of visual attention in dual-task situations. Psychological Review, 108, 393-434.

Lu, C.-H. (1997). Correspondence effects for irrelevant information in choice-reaction tasks: Characterizing the $\mathrm{S}-\mathrm{R}$ relations and the processing dynamics. In B. Hommel \& W. Prinz (Eds.), Theoretical issues in stimulus-response compatibility (pp. 85-117). Amsterdam: Elsevier, North-Holland.

Lu, C.-H., \& Proctor, R. W. (1995). The influence of irrelevant location information on performance: A review of the Simon and spatial Stroop effects. Psychonomic Bulletin \& Review, 2, 174-207.

Meiran, N. (2000). Modeling cognitive control in task-switching. Psychological Research, 63, 234-249.

Meyer, D. E., \& Kieras, D. E. (1997). A computational theory of executive control processes and human multiple-task performance: Part 1. Basic mechanisms. Psychological Review, 104, 3-65.

MülleR, H. J., \& RabbitT, P. M. (1989). Reflexive and voluntary orienting of visual attention: Time course of activation and resistance to interruption. Journal of Experimental Psychology: Human Perception \& Performance, 15, 315-330.

Posner, M. I. (1980). Orienting of attention. Quarterly Journal of Experimental Psychology, 32, 3-25.

Posner, M. I., \& Cohen, Y. (1984). Components of visual orienting. In H. Bouma \& D. G. Bouwhuis (Eds.), Attention and performance X: Control of language processes (pp. 531-556). Hillsdale, NJ: Erlbaum.

Pratt, J., \& Hommel, B. (2003). Symbolic control of visual attention:
The role of working memory and attentional control settings. Journal of Experimental Psychology: Human Perception \& Performance, 29, 835-845.

Reeves, A., \& Sperling, G. (1986). Attention gating in short-term visual memory. Psychological Review, 93, 180-206.

Tomasello, M., \& Call, J. (1997). Primate cognition. New York: Oxford University Press.

VIRZI, R. A., \& EGETH, H. E. (1985). Toward a translational model of Stroop interference. Memory \& Cognition, 13, 304-319.

Waszak, F., Hommel, B., \& Allport, A. (2003). Task-switching and long-term priming: Role of episodic stimulus task bindings in taskshift costs. Cognitive Psychology, 46, 361-413.

WASZAK, F., Hommel, B., \& AlLPort, A. (2005). Interaction of task readiness and automatic retrieval in task switching: Negative priming and competitor priming. Memory \& Cognition, 33, 595-610.

WeichSelgartner, E., \& SPERLING, G. (1987). Dynamics of automatic and controlled visual attention. Science, 238, 778-780.

\section{NOTES}

1. In trials in which a letter appeared twice, it was possible that even though the participant reported the copy that appeared prior to the target, it was erroneously scored as appearing after the target (which were the only cases we considered in our analysis). However, given the random letter selection, this merely added to the noise and could not create a systematic effect.

2. One might object that, overall, performance in Experiment 2 was no better than in Experiment 1, which seems to speak against the claim that the cue was easier to interpret. However, note that presenting the stream elements in (relatively dark) colors decreased their discriminability, which must have impaired performance. We thus attribute the fact that such impairments were hardly visible to the compensating effect of the cue manipulation.

(Manuscript received May 17, 2008; revision accepted for publication September 2, 2008.) 